\section{Crystallographic data deposition}

SIR - A formal discussion of the archival journal requirements for data deposition was held at the international seminar-cumschool on macromolecular crystallographic data at Calcutta, India, in November.

The current policy of the International Union of Crystallography (IUCr) is that on publication of a crystal structure determination of a macromolecule, the atomic parameters used or represented in the publication must be deposited in the Protein Data Bank. The deposition of structure amplitudes is recommended but not insisted on. The policy provides crystallographers with the option to delay the release of atomic parameters for one year and of structure amplitudes for up to four years from the date of publication. Participants strongly supported this policy and felt it should be strictly applied by the journals.

But recent developments in X-ray crystallographic experimental and refinement techniques and the huge expansion in computer power and networking make it necessary to review deposition arrangements. It was noted that the new validation procedures are much more effective but require the experimental structure amplitudes as well as the atomic parameters. In addition, the technical arrangements for deposition, analysis and validation of macromolecular crystal structures are now much easier.

We consider it vital for the macromolecular crystallographers to respond to these developments in their deposition practices. We recommend, therefore, that publication of macromolecular crystal structures should be accompanied by deposition of atomic parameters and also structure amplitudes. Among the many reasons identified for this practice, the following are critical.

(1) Rigorous validation of the structure determination results can be carried out only by using both atomic parameters and experimental structure amplitudes. It is important that journals should ensure that referees have sufficient information to prevent incorrect structures from being published.

(2) Archiving of these data will ensure that they are not lost. Numerous reports at this meeting of data being lost probably reflect a general problem in the crystallographic community.

Edward N. Baker (Member of $1 \mathrm{UCr}$ Executive Committee \& IUCr Commission on Biological Molecules), Department of Chemistry and Biochemistry, Massey University, Palmerston North, New Zealand Tom L. Blundell, ICRF Unit of Structural Molecular Biology, Department of Crystallography, Birkbeck College, London WC1E 7HX, UK Mamannamana Vijayan (Chairman of $\mathrm{IUCr}$ Commission on Biological Molecules), Molecular Biophysics Unit, Indian Institute of Science, Bangalore 560012, India
Eleanor Dodson (Member of IUCr Electronic Publishing Committee), Department of Chemistry, University of York, York YO1 5DD, UK Guy Dodson (Previous Chairman of $\mathrm{UCCr}$ Commission on Biological Molecules), Department of Chemistry, University of York, York YO1 5DD, UK

Gary I. Gilliland, Center for Advanced Research in Biotechnology, Rockville, Maryland 20850, USA

Joel L. Sussman (Head, Protein Data Bank), Departments of Biology and Chemistry, Brookhaven National Laboratory, Upton, New York 11973, USA \& Department of Structural Biology, Weizmann Institute of Science, Rehovot, Israel

See also Opinion, page 191.

\section{Panic and the Pill}

SIR - The Committee on the Safety of Medicines was indeed surprised by some aspects of the media's response to its advice on oral contraceptives (OCs) containing gestodene and desogestrel (Nature 377, $663 ; 1995)$. Our particular concern was that the lay press might publish misleading articles leading to a 'pill scare' with many women stopping using oral contraceptives altogether. The consequence would have been unplanned pregnancies, an increase in the abortion rate and the various risks that these entail. In fact, the lay media did an excellent job of reporting accurately the relevant brands, and of passing on our message that no one need stop taking OCs. In contrast to our expectations, it was the scientific press that failed to get the facts right.

Having correctly emphasized the importance of absolute risk, you quote a figure (1 in 200,000 per year) that is at least 30 times less than any estimate ever published for thromboembolism with OCs. The best estimate we have is that the excess risk associated with using OCs containing desogestrel or gestodene is about 15 thromboembolic events per 100,000 per year. Although the lay media quoted the correct figures for absolute risk, Nature was not alone in providing its readers with incorrect factual information. J. Guilleband in the British Medical Journal (311,1111-1112; 1995) managed to express the excess risk as 15 per 100,000 women. Because the risk remains fairly constant over time, and because women tend to use OCs for many years, Guilleband also seriously underestimated the absolute risk. Once your own estimate of risk is multiplied by 30 , the whole thrust of your leading article, that the Committee on the Safety of Medicines (CSM) acted unnecessarily, becomes untenable.

There are other serious inaccuracies. The implication that the committee and the
Medicines Control Agency failed to communicate with the relevant pharmaceutical companies is false; and the suggestion that they might be best placed to put out warnings that have important implications for public health is naive. It is also wrong to suggest that Professor Walter Spitzer might be in a better position to judge the position than the committee. That could be true only if he had information that he had not shared with us - and that is not the case. The CSM's advice was based on all the information available from three separate studies, and Spitzer's data were only part of the picture. Our advice also took into account considerations relating to contraceptive use and practice in the United Kingdom. Needless to say, I was surprised by Spitzer's actions; he presumably made the data available to us because he felt they might have important implications for public health. It is therefore difficult to understand why he objects when they were used for the purposes of protecting public health.

Finally, I find your statement that "sudden announcements... are almost always likely to cause panic" quite extraordinary. The press notices emphasized that there was no need to panic, and this was clearly explained on British television. How on Earth can an announcement like this be made gradually?

\section{Michael D. Rawlins}

(Chairman)

Committee on the Safety of Medicines,

Market Towers,

1 Nine Elms Lane,

London SW8 5NQ, UK

\section{Games people play}

SIR - Sally Lehrman errs in saying that the University of California at San Francisco (UCSF) rivals Stanford University "on the football field" (Nature 378, 529; 1995). UCSF has never had a football team. Stanford cherishes its football rivalry with the University of California, Berkeley, known also as 'CAL' and 'The Golden Bears', the latter in reference to the California Grizzly, totem animal of CAL and the State of California. Stanford shows its preoccupation by a toll-free telephone number 1-800-BEAT CAL. The corresponding UC number is 1$800-$ GO BEARS. The University of California, Los Angeles, also has a football team that frequently trounces Stanford.

\section{Thomas H. Jukes}

University of California, Berkeley, Berkeley, California 94720, USA

\section{Correspondence}

Letters submitted for Correspondence should be typed, double-spaced, on one side of the paper only, or e-mailed to nature@nature.com 